\title{
Adaptive optical phase estimation
}

\author{
D. Nakane ${ }^{1}$, T. A. Wheatley ${ }^{1,2,3}$, D. W. Berry ${ }^{4}$, H. Yonezawa ${ }^{1}$, H. Arao ${ }^{1}$, \\ D. T. Pope ${ }^{5}$, T. C. Ralph ${ }^{2,6}$ H. M. Wiseman ${ }^{2,7}$, E. H. Huntington ${ }^{2,3}$ \\ and A. Furusawa ${ }^{1}$ \\ ${ }^{1}$ Department of Applied Physics and Quantum-Phase Electronics Center, School of Engineering. \\ The University of Tokyo, 7-3-1 Hongo, Bunkyo-ku, Tokyo, Japan \\ ${ }^{2}$ Centre for Quantum Computer Technology, Australian Research Council \\ ${ }^{3}$ School of Engineering and Information Technology, University college, \\ The University of New South Wales, Canberra 2600, ACT, Australia \\ ${ }^{4}$ Institute for Quantum Computing, University of Waterloo, Waterloo, N2L 3G1, ON, Canada \\ ${ }^{5}$ Perimeter Institute, Waterloo, ON, Canada \\ ${ }^{6}$ Department of Physics, University of Queensland, Brisbane 4072, QLD, Australia \\ ${ }^{7}$ Centre for Quantum Dynamics, Griffith University, Brisbane 4111, QLD, Australia \\ nakane@alice.t.u-tokyo.ac.jp
}

\begin{abstract}
We experimentally performed adaptive phase estimation using time-symmetric quantum smoothing for a stochastically varying phase on continuous wave coherent beam. We demonstrate better accuracy than conventional methods.

(C) 2010 Optical Society of America

OCIS codes: (120.5050) Phase measurement; (120.1088) Adaptive interferometry
\end{abstract}

Phase estimation of an electromagnetic field is applicable to various fields, from optical communication to gravitational wave detection. In those applications, accuracy of the phase estimation is essential. Unfortunately phase cannot be measured directly [1], and should be estimated by other variables such as quadrature amplitudes of the field. Conventional methods are simultaneous measurements of conjugate variables (heterodyne or dual homodyne measurements) in which extra noise from the measurement back-action is inevitably added. Adaptive homodyne measurement $[2,3]$ is an alternative way to measure phase in which a single homodyne detector and phase-locked loop are used. Adaptive homodyne measurement enables better phase estimation than the conventional methods. However, the accuracy of phase estimation depends on both measurement scheme and estimation technique. If we allow time-symmetric estimation, which is based on the data taken before and after a particular time, the estimate can be even more precise. In this presentation we demonstrate adaptive phase estimation for continuous wave light, and present the first experimental demonstration of time-symmetric quantum smoothing [4].

Fig.1 shows schematics of our experiments. We impose Ornstein-Uhlenbeck (O-U) noise on a signal beam. The signal phase $\varphi(t)$ is varied as

$$
\varphi(t)=\kappa \int_{-\infty}^{t} e^{\lambda(s-t)} d W(s),
$$

where $d W(s)$ is a Wiener increment. In our experiment, we perform dual homodyne measurement to compare with adaptive homodyne measurement. In dual homodyne measurement, two orthogonal quadratures $x$ and $p$ are simultaneously measured by two homodyne detections, as shown in Fig. 1(a). Using two homodyne photocurrents $I_{x}(t)$ and $I_{p}(t)$, an instantaneous phase estimate at time $t$ is obtained as $\theta(t)=\arg \left(I_{x}(t)+\right.$ $\left.i I_{p}(t)\right)$. This instantaneous estimate $\theta(t)$ is the best estimate with the data taken in time interval $[t, t+d t)$ [5]. The final phase estimate is obtained through filtering or smoothing this instantaneous estimate $\theta(t)$. In Fig. 1(b), adaptive homodyne measurement is shown. Homodyne photocurrent $I(t)$ is fed back to local oscillator (LO) phase $\Phi(t)$ through a feedback filter so that the LO phase locks to the signal phase plus $\pi / 2$, where homodyne detection is most sensitive. That is, $\hat{\varphi}(t)=\Phi(t)-\pi / 2$ is a rough estimate of signal phase $\varphi(t)$. Note that we use a simple linear low-pass filter for the feedback, then $\hat{\varphi}(t)$ is a weighted average of the instantaneous phase estimate $\theta(t)[5]$.

Here we apply two different phase estimation methods, i.e., filtering and time-symmetric smoothing. Quantum filtering is performed by applying a linear low-pass filter to the instantaneous phase estimate $\theta(t)[5]$,

$$
\Theta_{-}(t)=\chi \int_{-\infty}^{t} e^{\chi(s-t)} \theta(s) d s
$$

where $\Theta_{-}(t)$ is the estimate of the filtering and $\chi$ is a cut-off frequency of the low-pass filter. Note that, in the case of adaptive homodyne, we use $\hat{\varphi}(t)$ instead of $\theta(t)$. The filtered estimate at time $t$ only uses the data taken before the time $t$. If there is no need for a real time phase estimate, we can make a more accurate estimate using time-symmetric smoothing. In this case, the phase estimate at time $t$ is based on the 


\section{JThF4.pdf}

(a)

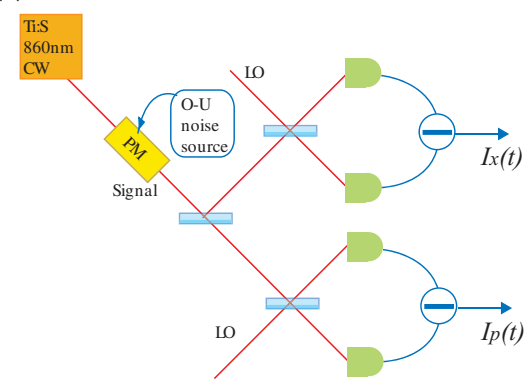

(b)

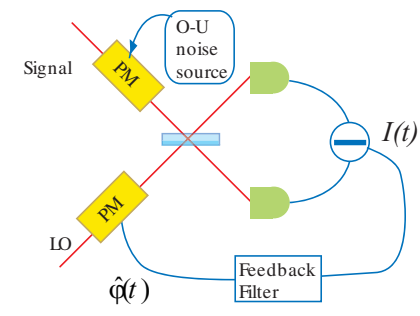

Fig. 1. Schematics of the experiment. Continuous wave Titanium Sapphire laser at $860 \mathrm{~nm}$ is used. PM stands for a phase modulator. (a) Dual homodyne measurement. (b) Adaptive homodyne measurement.

date taken both before $t$ and after $t$. We denote a phase estimate based on measurements after $t$ by $\Theta_{+}(t)$. $\Theta_{+}(t)$ is obtained by performing the same low-pass filter backward on the instantaneous phase estimate $\theta(t)$. Then the time-symmetric estimate is obtained by averaging the forward and backward estimates as $\Theta(t)=\left(\Theta_{-}(t)+\Theta_{+}(t)\right) / 2$. The accuracy of the estimation is defined by $\Delta \Theta^{2}=\left\langle(\Theta(t)-\varphi(t))^{2}\right\rangle$, and the theoretically calculated values for $\mathrm{O}-\mathrm{U}$ process are as follows.

$$
\Delta \Theta_{-}^{2}(\chi)=\left\{\begin{array}{ll}
\frac{\kappa^{2}}{2(\chi+\lambda)}+\frac{\chi}{8 N} & \text { (Adaptive filter) } \\
\frac{\kappa^{2}}{2(\chi+\lambda)}+\frac{\chi}{4 N} & \text { (Dual filter) }
\end{array}, \Delta \Theta^{2}(\chi)=\left\{\begin{array}{ll}
\frac{\kappa^{2}(\chi+2 \lambda)}{4(\chi+\lambda)^{2}}+\frac{\chi}{16 N} & \text { (Adaptive smoothing) } \\
\frac{\kappa^{2}(\chi+2 \lambda)}{4(\chi+\lambda)^{2}}+\frac{\chi}{8 N} & \text { (Dual smoothing) }
\end{array} .\right.\right.
$$

Fig. 2 shows phase estimation in the time domain at an optimal parameter $\chi$. For comparison, we show two experimental results, that is, dual homodyne estimation using filtering and adaptive homodyne estimation using time-symmetric smoothing. The superiority of adaptive smoothing is quite obvious. Fig. 3 shows the phase variance $\Delta \Theta^{2}$ at variable parameter $\chi$ with various phase estimation schemes. The variances for optimum $\chi$ are 0.033 for adaptive smoothing, 0.059 for adaptive filtering, 0.044 for dual smoothing, and 0.068 for dual filtering. The experimental results show good agreements with the theoretical curves. Moreover the phase variances of the adaptive schemes are clearly below the standard quantum limit set by an ideal dual homodyne measurement using filtering. In this presentation we will present these experimental results.
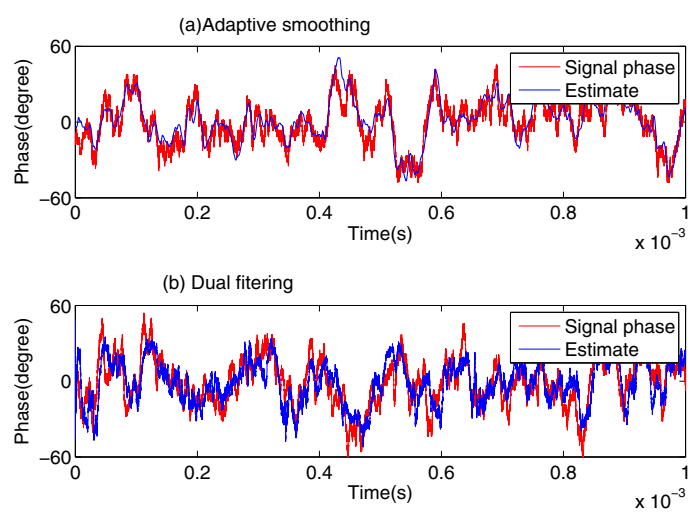

Fig. 2. Phase estimation in the time domain. (a) Adaptive homodyne estimation using time-symmetric smoothing. (b) Dual homodyne estimation using filtering.

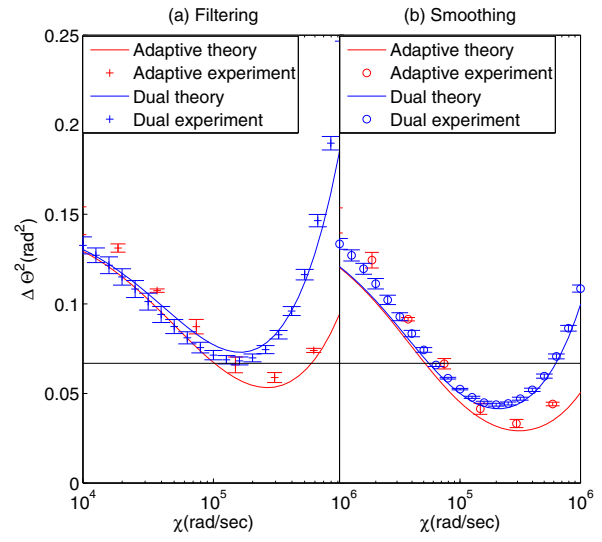

Fig. 3. Phase variance as a function of $\chi$. (a) Filtering (b) Smoothing. The black line is SQL set by ideal dual homodyne. Parameters are: $\kappa=1.4 \times 10^{2}, \lambda=6.2 \times 10^{4}, N=$ $1.3 \times 10^{6}$ for dual, $1.4 \times 10^{6}$ for adaptive.

[1] U. Leonhardt, J. A. Vaccaro, B. Böhmer, and H. Paul, Phys. Rev. A 51, 84 (1995).

[2] H. M. Wiseman, Phys. Rev. Lett. 75, 4587 (1995).

[3] M. A. Armen, J. K. Stockton, A. C. Doherty, and H. Mabuchi, Phys. Rev. Lett. 89, 13362 (2002).

[4] M. Tsang, Phys. Rev. Lett. 102, 250403 (2009).

[5] D. W. Berry and H. W. Wiseman Phys. Rev. A 65, 043803 (2002). 